\title{
Front Matter: Volume 10143
}

, "Front Matter: Volume 10143," Proc. SPIE 10143, Extreme Ultraviolet (EUV) Lithography VIII, 1014301 (5 May 2017); doi: 10.1117/12.2279234

SPIE. Event: SPIE Advanced Lithography, 2017, San Jose, California, United States 


\title{
PROCEEDINGS OF SPIE
}

\section{Extreme Ultraviolet (EUV) Lithography VIII}

\author{
Eric M. Panning \\ Kenneth A. Goldberg \\ Editors
}

27 February - 2 March 2017

San Jose, California, United States

Sponsored by

SPIE

Cosponsored by

ASML, Inc. (United States)

Published by

SPIE 
The papers in this volume were part of the technical conference cited on the cover and title page. Papers were selected and subject to review by the editors and conference program committee. Some conference presentations may not be available for publication. Additional papers and presentation recordings may be available online in the SPIE Digital Library at SPIEDigitallibrary.org.

The papers reflect the work and thoughts of the authors and are published herein as submitted. The publisher is not responsible for the validity of the information or for any outcomes resulting from reliance thereon.

Please use the following format to cite material from these proceedings:

Author(s), "Title of Paper," in Extreme Ultraviolet (EUV) Lithography VIII, edited by Eric M. Panning, Kenneth A. Goldberg, Proceedings of SPIE Vol. 10143 (SPIE, Bellingham, WA, 2017) Seven-digit Article CID Number.

ISSN: 0277-786X

ISSN: 1996-756X (electronic)

ISBN: 9781510607378

ISBN: 9781510607385 (electronic)

Published by

SPIE

P.O. Box 10, Bellingham, Washington 98227-0010 USA

Telephone +1 3606763290 (Pacific Time) · Fax +1 3606471445

SPIE.org

Copyright @ 2017, Society of Photo-Optical Instrumentation Engineers.

Copying of material in this book for internal or personal use, or for the internal or personal use of specific clients, beyond the fair use provisions granted by the U.S. Copyright Law is authorized by SPIE subject to payment of copying fees. The Transactional Reporting Service base fee for this volume is $\$ 18.00$ per article (or portion thereof), which should be paid directly to the Copyright Clearance Center (CCC), 222 Rosewood Drive, Danvers, MA 01923. Payment may also be made electronically through CCC Online at copyright.com. Other copying for republication, resale, advertising or promotion, or any form of systematic or multiple reproduction of any material in this book is prohibited except with permission in writing from the publisher. The CCC fee code is 0277-786X/17/\$18.00.

Printed in the United States of America.

Publication of record for individual papers is online in the SPIE Digital Library.

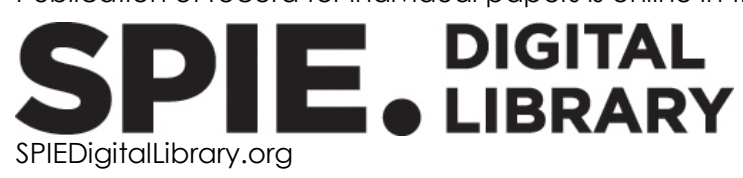

Paper Numbering: Proceedings of SPIE follow an e-First publication model, with papers published first online and then in print. Papers are published as they are submitted and meet publication criteria. A unique citation identifier (CID) number is assigned to each article at the time of the first publication. Utilization of CIDs allows articles to be fully citable as soon as they are published online, and connects the same identifier to all online, print, and electronic versions of the publication. SPIE uses a seven-digit CID article numbering system in which:

- The first five digits correspond to the SPIE volume number.

- The last two digits indicate publication order within the volume using a Base 36 numbering system employing both numerals and letters. These two-number sets start with 00, 01, 02, 03, 04, $05,06,07,08,09,0 A, 0 B \ldots$ OZ, followed by 10-1Z, 20-2Z, etc. The CID Number appears on each page of the manuscript. The complete citation is used on the first page of the manuscript. 


\title{
Contents
}

\author{
ix Authors \\ xiii Conference Committee
}

\section{KEYNOTE SESSION}

1014306 Progress in EUV lithography toward manufacturing (Keynote Paper) [10143-2]

EUV MATERIALS I: METAL-BASED EUV RESISTS: JOINT SESSION WITH CONFERENCES 10146 AND 10143

$1014308 \quad$ Nanoparticle photoresist studies for EUV lithography [10143-4]

1014309 Advanced development techniques for metal-based EUV resists [10143-5]

EUV MATERIALS II: FUNDAMENTALS I: JOINT SESSION WITH CONFERENCES 10146 AND 10143

10143 OA Absorption coefficient and exposure kinetics of photoresists at EUV [10143-6]

10143 OB Correlation of experimentally measured atomic scale properties of EUV photoresist to modeling performance: an exploration [10143-7]

INTEGRATION

10143 OD Enabling sub-10nm node lithography: presenting the NXE:3400B EUV scanner (Invited Paper) [10143-9]

10143 OE Line-edge roughness performance targets for EUV lithography [10143-10]

10143 OF Mix-and-match considerations for EUV insertion in N7 HVM [10143-11]

10143 OG The future of EUV lithography: enabling Moore's Law in the next decade [10143-12]

$10143 \mathrm{OH} \quad$ SAQP and EUV block patterning of BEOL metal layers on IMEC's iN7 platform [10143-13]

$10143 \mathrm{Ol} \quad$ Modeling EUVL patterning variability for metal layers in $5 \mathrm{~nm}$ technology node and its effect on electrical resistance [10143-14] 
EUV MASK INSPECTION AND IMAGING: JOINT SESSION WITH CONFERENCES 10143 AND 10145

10143 OJ Actinic review of EUV masks: performance data and status of the AIMS EUV system [10143-15]

10143 OK Printability and actinic AIMS review of programmed mask blank defects [10143-16] EUV OPTICS AND PELLICLE

$10143 \mathrm{OL} \quad$ Novel membrane solutions for the EUV pellicle: better or not? [10143-17]

10143 OM Impact of tool design on defect detection sensitivity for EUV actinic blank inspection [10143-18]

10143 ON High-NA metrology and sensing on Berkeley MET5 [10143-19]

10143 OP Investigating surface structures by EUV scattering [10143-21]

RESIST ADVANCES AND INTEGRATION

$101430 Q \quad$ Improvements in resist performance towards EUV HVM (Invited Paper) [10143-22]

10143 OR Exploring the readiness of EUV photo materials for patterning advanced technology nodes [10143-23]

10143 OT State-of-the-art EUV materials and processes for the 7nm node and beyond [10143-25]

10143 OU High-volume manufacturing compatible dry development rinse process (DDRP): patterning and defectivity performance for EUVL [10143-26]

10143 OV Sensitivity enhancement of the high-resolution xMT multi-trigger resist for EUV lithography [10143-27]

\section{RESIST MODELING}

10143 OW Mechanisms of EUV exposure: electrons and holes [10143-28]

10143 OY Driving down defect density in composite EUV patterning film stacks [10143-30]

$101430 Z \quad$ Lithographic stochastics: beyond 3\% [10143-31]

MASKS I

$1014310 \quad$ Reducing EUV mask 3D effects by alternative metal absorbers [10143-32]

iv 
$1014311 \quad$ N7 dark field two-bar in 0.33NA EUVL: Mitigation of CD Bossung tilts caused by strong coupling between the feature's primary and 1st self-image [10143-33]

1014312 Investigation of alternate mask absorbers in EUV lithography [10143-34]

1014313 Vote-taking for EUV lithography: a radical approach to mitigate mask defects [10143-35]

1014314 Reticle enhancement techniques toward iN7 metal2 [10143-36]

MASKS II

1014315 Considerations for pattern placement error correction toward 5nm node [10143-37]

1014316 Enabling full field physics based OPC via dynamic model generation [10143-39]

1014317 Rigorous 3D electromagnetic simulation of ultrahigh efficiency EUV contact-hole printing with chromeless phase shift mask [10143-40]

\section{PATTERNING I}

1014318 Single exposure EUV patterning of BEOL metal layers on the IMEC iN7 platform (Invited Paper) [10143-41]

1014319 Integrated approach to improving local CD uniformity in EUV patterning [10143-42]

$101431 \mathrm{~A}$ Comprehensive analysis of line-edge and line-width roughness for EUV lithography [10143-43]

10143 1C New methodologies for lower-K1 EUV OPC and RET optimization [10143-45]

10143 1D Ultrathin EUV patterning stack using polymer brush as an adhesion promotion layer [10143-46]

\section{PATTERNING II}

10143 1E Compact 2D OPC modeling of a metal oxide EUV resist for a 7nm node BEOL layer [10143-47]

10143 1F Minimizing wafer overlay errors due to EUV mask non-flatness and thickness variations for N7 production [10143-48]

$101431 \mathrm{G}$ Single-expose patterning development for EUV lithography [10143-49]

$101431 \mathrm{H}$ Single-nm resolution approach by applying DDRP and DDRM [10143-50] 
SOURCE

$101431 \mathrm{~J} \quad$ Performance of 250W high-power HVM LPP-EUV source [10143-52]

POSTERS: INSPECTION

$101431 \mathrm{~N} \quad$ First light at EBL2 [10143-56]

1014310 RESCAN: an actinic lensless microscope for defect inspection of EUV reticles [10143-57]

$101431 \mathrm{P} \quad$ Arc-shaped slit effect of EUV lithography with anamorphic high-NA system in terms of critical dimension variation [10143-58]

$101431 Q$ A two-step method for fast and reliable EUV mask metrology [10143-59]

POSTERS: MASKS AND OPTICS/PELLICLE

10143 is Monte Carlo sensitivity analysis of EUV mask reflectivity and its impact on OPC accuracy [10143-61]

$101431 \mathrm{~T} \quad$ A study on EUV reticle surface molecular contamination under different storage conditions in a HVM foundry fab [10143-62]

$101431 U$ CD error caused by aberration and its possible compensation by optical proximity correction in extreme-ultraviolet lithography [10143-63]

10143 1V Impact of non-uniform wrinkles for a multi-stack pellicle in EUV lithography [10143-64]

10143 IW Image-based pupil plane characterization for anamorphic lithography systems [10143-89]

POSTERS: PRINTING

$101431 Y \quad$ Contribution of EUV mask CD variability on LCDU [10143-66]

1014312 EUV process improvement with novel litho track hardware [10143-67]

1014320 Impact of EUV SRAF on Bossung tilt [10143-68]

$1014321 \quad$ 2D self-aligned via patterning strategy with EUV single-exposure in $3 \mathrm{~nm}$ technology [10143-90]

POSTERS: RESIST

1014322 A study on enhancing EUV resist sensitivity [10143-69]

1014323 Impact of acid statistics on EUV local critical dimension uniformity [10143-70]

vi 
1014324 Influence of post exposure bake time on EUV photoresist RLS trade-off [10143-71]

1014325 Extreme ultraviolet patterning of tin-oxo cages [10143-72]

1014326 Technology for defectivity improvement in resist coating and developing process in EUV lithography process [10143-73]

1014327 In-situ measurement of outgassing generated from EUV resist including metal oxide nanoparticles during electron irradiation [10143-74]

1014328 Irresistible Materials multi-trigger resist: the journey towards high volume manufacturing readiness [10143-75]

1014329 Simulation and experimentation of PSCAR chemistry for complex structures [10143-76]

$101432 \mathrm{~B}$ Optimization of stochastic EUV resist models parameters to mitigate line edge roughness [10143-78]

$101432 \mathrm{C}$ Contrast curves for low energy electron exposures of an EUV resist in a scanning electron microscope [10143-79]

10143 2E Computational approach on PEB process in EUV resist: multi-scale simulation [10143-91]

POSTERS: SOURCE

$101432 \mathrm{G}$ Key components technology update of the 250W high-power LPP-EUV light source [10143-83]

$1014321 \quad$ Study of Sn removal by surface wave plasma for source cleaning [10143-85]

$101432 \mathrm{~J} \quad$ Background pressure effects on EUV source efficiency and produced debris characteristics [10143-86]

$101432 \mathrm{~K} \quad$ Improvement of power, efficiency, and cost of ownership in the tin LPP EUV source [10143-88] 
Proc. of SPIE Vol. 10143 1014301-8

Downloaded From: https://www.spiedigitallibrary.org/conference-proceedings-of-spie on 26 Apr 2023 Terms of Use: https://www.spiedigitallibrary.org/terms-of-use 


\section{Authors}

Numbers in the index correspond to the last two digits of the seven-digit citation identifier (CID) article numbering system used in Proceedings of SPIE. The first five digits reflect the volume number. Base 36 numbering is employed for the last two digits and indicates the order of articles within the volume. Numbers start with 00, 01, 02, 03, 04, 05, 06, 07, 08, 09, 0A, 0B...0Z, followed by 10-1Z, 20-2Z, etc.

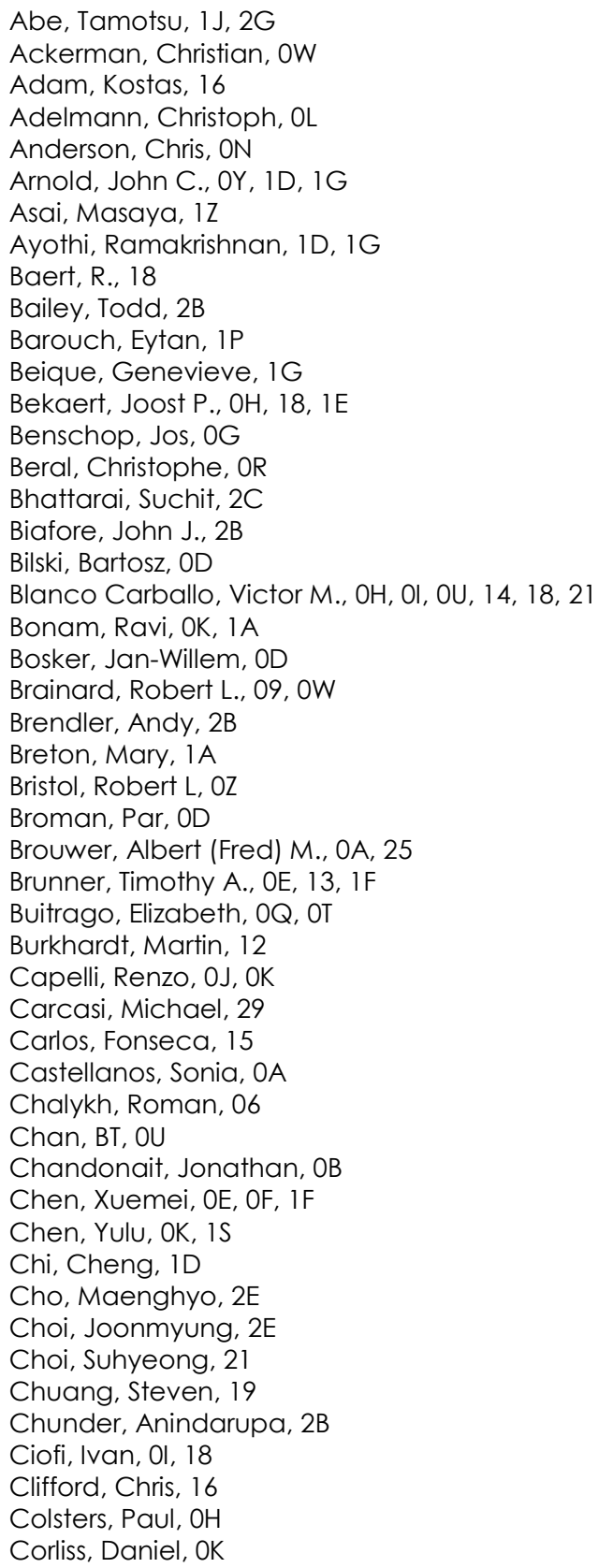

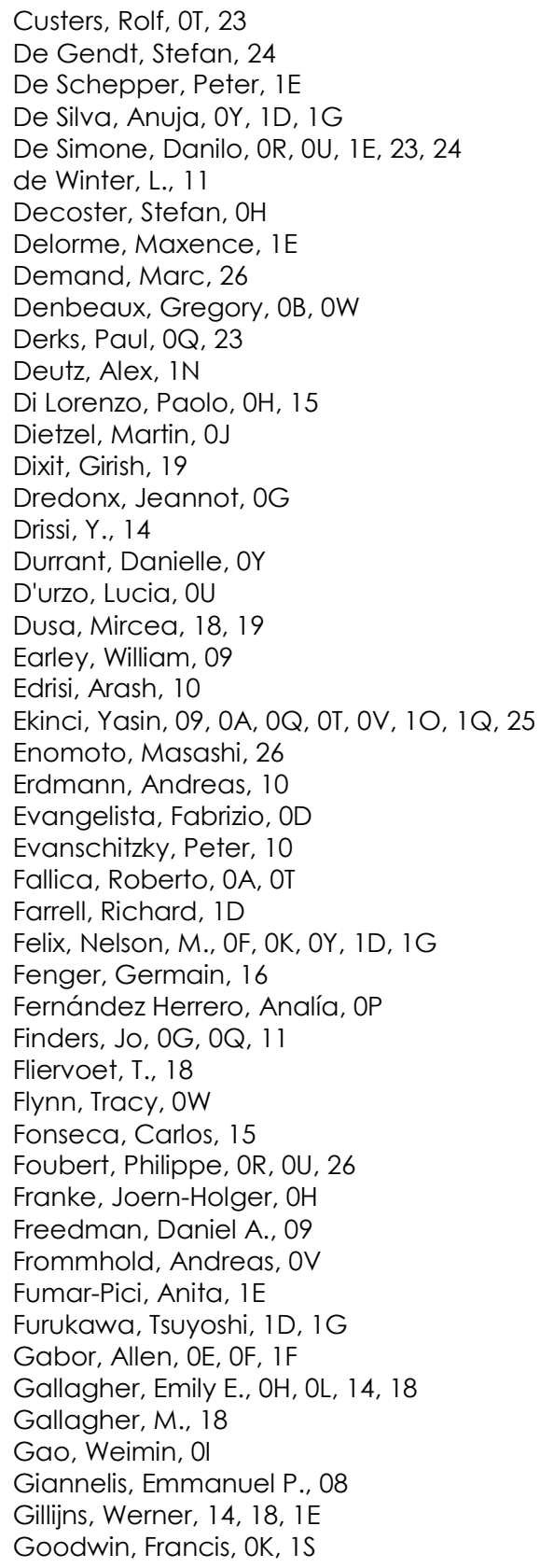


Greer, Michael, 1 E

Grzeskowiak, Steven, OB, OW

Gullikson, Eric, 1S

Guo, Jing, 1D

Haase, Anton, OP

Haitjema, Jarich, OA, 25

Halder, S., 18

Hamieh, Bassem, $1 G$

Han, Geng, 13

Han, Seulgi, 1G

Hara, Arisa, 15

Harada, Tetsuo, 22

Harm, Greg, 19

Harumoto, Masahiko, $1 \mathrm{Z}$

Heil, Tilmann, OG

Helfenstein, Patrick, 10, 1Q

Hellweg, Dirk, OJ, OK

Hendrickx, Eric, $\mathrm{OH}, \mathrm{Ol}, 10,18$

Hermans, Jan, 19

Hetel, Iulian, OU

Hetzer, Dave, OY

Higgins, Craig, $\mathrm{OE}$

Hoefnagels, Rik, OQ, OT, 23

Hollemans, Christiaan, $1 \mathrm{~N}$

Hollink, T., 18

Hontake, Koichi, OY

Hooker, Kevin, $1 \mathrm{C}$

Hori, Tsukasa, 1 J, 2G

Hosler, Erik, OF

Hotalen, Jodi, 09

Hsu, Stephen, 11, 20

Huang, Huai, IA

Hubbard, Alex, OY

Huli, Lior, OY

Huyghebaert, Cedric, OL

Hwang, Jeong-Gu, $1 \mathrm{U}$

Hwang, Myungsoo, 06

Ichinomiya, Hiroshi, 26

Irmscher, Mathias, 10

Iseki, Tomohiro, 29

Jasper, Hans, OD

Jeon, Jinho, 06

Jeong, Changyoung, 2E

Jiang, Jing, 23

Johnson, Richard, OF, OY

Kadoi, Mikio, 27

Kaiser, Winfried, OG

Kamei, Yuya, 26

Kamohara, Itaru, 01

Kandel, Yudhishthir, OB

Kaneyama, Koji, $1 \mathrm{Z}$

Kasahara, Kazuki, 08

Kawakami, Shinichiro, OY, 26

Kawasuji, Yasufumi, 1 J, 2G

Kazarian, Aram, 1C

Kazazis, Dimitrios, 25

Kerkhof, Peter, $1 \mathrm{~N}$

Kiers, Ton, $\mathrm{OH}, 15$

Kim, Byung-Hun, $1 \mathrm{U}$
Kim, Guk-Jin, 1P, 1U, 1V

Kim, Heebom, 2E

Kim, Hocheol, 06

Kim, Hoyeon, 06

Kim, Hyunwoo, 06

Kim, In-Seon, IP, IU, IV

Kim, Insung, 06

Kim, Jungyeop, 06

Kim, Min-Su, IV

Kim, Muyoung, 2E

Kim, R. Ryoung-Han, OH, Ol, 14, 18, 21

Kim, Seong-Sue, 06

Kneer, Bernhard, OG

Koch, Markus, OJ

Kocsis, Michael, $1 \mathrm{E}$

Kodama, Takeshi, 1 J, 2 G

Koike, Kyohei, 15

Kosma, Vasiliki, 08

Koster, Norbert, $1 \mathrm{~N}$

Krabbendam, Peter, OG

Krysak, Marie E., $\mathrm{OZ}$

Kubis, Michael, 19

Kutrzeba Kotowska, Bogumila, $0 \mathrm{H}, 18$

Kye, Jongwook, is

Lam, Michael, 16

Larivière, Stéphane, $\mathrm{OH}, 18$

Last, Thorsten, OD, 11

Latypov, Azat, 2B

Laubis, Christian, OP, 10

Lazzarino, Frederic, $\mathrm{OH}, \mathrm{OR}, 18$

Lee, Byunghoon, 2E

Lee, Donggun, 06

Lee, Jae Uk, OL, 21

Lee, Joe, $1 G$

Lee, Seungkoo, 06

Lee, Siyong, 06

Lee, Sook, $1 \mathrm{E}$

Lemley, Corey, OY

Lenderink, Egbert, OD

Leray, Philippe, $\mathrm{OH}, 18$

Levasier, Leon, OD

Levinson, Harry J., 1Y, 2B

Levinson, Zac, IW

Liang, Andrew, 19

Liang, Chen-Wei, 19

Liu, Chi-Chun (Charlie), 1A, ID

Loopstra, Erik, OG

Lucas, Kevin, 1C

Luong, Kim Vu, 10

Lyons, Adam, $1 \mathrm{E}$

Mack, Chris A., OE

Mallik, A., 18

Mangat, Pawitter, OK, IT

Mao, Ming, OH, OR, 18

Marokkey, Sajan, OB

Maslow, Mark John, $\mathrm{OH}, 15$

Matham, Shravan, OK

Matsumoto, Yoko, 27

Matsunaga, Koichi, OY 
McClelland, Alexandra L., OV, 28

McGeoch, Malcolm W., 2K

McIntyre, Greg, OH, 14, 18

Meeuwissen, Marieke, 0Q, OT, 23

Meiling, Hans, OG

Meli, Luciana, OY, 1D, IG

Melvin, Lawrence S., OB, Ol

Meyer-Ilse, Julia, $1 \mathrm{~S}$

Meyers, Sheldon, OF

Migura, Sascha, OG

Mika, Niclas, OG

Minami, Yoichi, 27

Minekawa, Yukie, 29

Miyakawa, Ryan, ON

Mizoguchi, Hakaru, 1 J, 2G

Mochi, Iacopo, 0T, 10, 1Q

Mohacsi, Istvan, 10

Molkenboer, Freek, $1 \mathrm{~N}$

Montgomery, Warren, 28

Moon, Junghwan, 2E

Muilwijk, Pim, $1 \mathrm{~N}$

Mulckhuyse, Wouter, $1 \mathrm{~N}$

Murphy, Michael, 09

Murray, Cody, OY

Muthinti, Raja, 1A

$\mathrm{Na}$, Jihoon, 06

Naasz, Sandra, 10

Nafus, Kathleen, 26

Nagahara, Seiji, 29

Nagai, Tomoki, 29

Nakagawa, Hisashi, 29

Nakajima, Makoto, $1 \mathrm{H}$

Nakarai, Hiroaki, 1 J, 2G

Nam, Seok-Woo, 06

Narasimhan, Amrit, OW

Naruoka, Takehiko, 29

Naulleau, Patrick P., OM, ON, 17, 20, 2 C

Natori, Sakurako, 15

Neureuther, Andrew R., OM, 17, 20, 2 C

Nijland, Björn, $1 \mathrm{~N}$

No, Hee-Ra, $1 \mathrm{U}$

Nowak, Krzysztof M., 1J, 2G

Ober, Christopher K., 08

Odent, Jeremy, 08

Oh, Hye-Keun, IP, IU, IV

Oh, Taehwan, $1 \mathrm{G}$

Okamoto, Takeshi, $2 \mathrm{G}$

Okazaki, Shinji, 1J, 2G

Oostdijck, Bastiaan, $1 \mathrm{~N}$

Oshima, Akihiro, 29

Oyama, Kenichi, 15

Ozlem, Melih, 13

Painter, Benjamin, OB

Panici, Gianluca, 21

Paolillo, S., 18

Park, Changmin, 06

Park, Jin-Goo, $1 \mathrm{~V}$

Park, Jinhong, 06

Park, Joo-On, 06
Patlolla, Raghuveer, $1 \mathrm{~A}$

Peeters, Rudy, OD

Perlitz, Sascha, OJ, OK

Petrillo, Karen, OY, $1 G$

Pflüger, Mika, OP

Philipsen, Vicky, OI, 10

Pieczulewski, Charles, 1 Z

Pirati, Alberto, OG

Pistor, Thomas V., 17

Pollentier, Ivan, $0 \mathrm{~L}$

Popescu, Carmen, OV

Qerimi, Dren, 2l

Qi, Zhengqing John, 1S, IY

Raghavan, Praveen, OU

Raghunathan, Ananthan, 16

Rajendran, Rajeev, 10, 1Q

Raley, Angelique, 1D

Rankin, Jed, OK, 13, 1F, 1S, 1Y

Reddy, Sirish, 19

Reuter, Christian, 10

Rio, David, 19, 1E

Rispens, Gijsbert, OQ, OT, 23

Robinson, Alex P. G., OV, 28

Roth, John, OV, 28

Rusu, P., 18

Ruzic, David N., 2l

Saad, Yves, 0 I

Saitou, Takashi, 1 J, 2G

Sakamoto, Rikimaru, $1 \mathrm{H}$

Samudrala, Pavan, OF

Saulnier, Nicole, 1A, $1 G$

Sayan, Safak, OU

Schmidt, Kristin, 1D

Scholze, Frank, OP, 10

Sekiguchi, Atsushi, 22, 27

Seshadri, Indira, 1A, 1D, $1 G$

Shamma, Nader, 19

Shearer, Jefferey C., IA, IG

Shehzad, Atif, OR

Sherazi, Y., 18

Sherwin, Stuart, 17

Shibayama, Wataru, $1 \mathrm{H}$

Shigaki, Shuhei, $1 \mathrm{H}$

Shin, Youngsoo, 21

Shiozawa, Takahiro, 26

Shiraishi, Gosuke, 29

Shiraishi, Yutaka, 1 J, 2 G

Shite, Hideo, 26

Sieg, Stuart, $1 \mathrm{~A}$

Singh, Lovejeet, 1D, IG

Singh, SherJang, IT

Singhal, Akhil, 19

Sizyuk, Tatyana, 2J

Smith, Bruce W., IW

Socha, Robert, 20

Soltwisch, Victor, OP

Souriau, Laurent, 10

Stamm, Uwe, OG

Stoeldraijer, Judon, OG 
Stokes, Harold, 1 Z

Stowers, Jason K., $1 \mathrm{E}$

Sun, Lei, OE, 1G, 1S, IY

Tagawa, Seiichi, 29

Takahashi, Seiji, 27

Takeda, Satoshi, $1 \mathrm{H}$

Tan, L. E., 14, 18

Tan, Samantha, 19

Tanaka, Hiroshi, 1 J, 2G

Tanaka, Yuji, $1 Z$

Tasdemir, Zuhal, OT

Taylor, Ron, $1 \mathrm{~T}$

te Sligte, Edwin, $1 \mathrm{~N}$

Thuering, Bernd, OG

Timmermans, Marina, OL

Timoshkov, Vadim, $\mathrm{OH}, 15$

Tran, Timothy, 19

Trivkovic, D., 14, 18

Troost, Kars, OG

Truang, Hoa, 1D

Turley, Christina, IF

Tuttle, Josh, 1C

Ure, David, 28

van Adrichem, P., 11

van Ballegoij, Rob, OG

van de Kerkhof, Mark, OD, 11

van de Kruijs, Robbert W. E., 10

Van Den Heuvel, Dieter, OR

van der Walle, Peter, IN

van Dijk, Joep, $\mathrm{OH}$

van Es, Roderik, OD

van Oosten, Anton, $0 Q$

van Putten, Michel, $1 \mathrm{~N}$

van Schoot, Jan, OG

van Schravendijk, Bart, 19

van Setten, Eelco, OG

Vandenberghe, Geert, OR, OU

Vandereyken, Jelle, 19

Vanelderen, Pieter, OU

Verduijn, Erik, OK, 13

Vesters, Yannick, OR, 24

Viatkina, Katja, 19

Vockenhuber, Michaela, 09, 0Q, 0T, 25

Wallow, Thomas I., $1 \mathrm{E}$

Wan, D., 18

Wang, Yow-Gwo, 0M, 20

Ward, Brandon, 19

Watanabe, Takeo, 22, 27

Watanabe, Yukio, 1 J, 2G

Westerhout, Jeroen, $1 \mathrm{~N}$

Wiaux, Vincent, 0

Wise, Rich, 19

Wittebrood, Friso, $\mathrm{OH}, 11,18$

Wood, Obert R., OK, 13, 1S, IT

Wu, Lianjia, OA

Wuister, Sander, $0 Q$

Xiao, Guangming, 1C

$\mathrm{Xu}$, Dongbo, 10
$\mathrm{Xu}$, Hong, 08

Yaegashi, Hidetami, 15

Yamada, Tsuyoshi, 1J

Yamato, Masatoshi, 15

Yamauchi, Shohei, 15

Yamazaki, Taku, 1 J, 2G

Yan, Qiliang, OB

Yanagida, Tatsuya, $1 \mathrm{~J}$

Yatzor, Brett, $1 \mathrm{~T}$

Yeung, Michael, 1P, 1V

Yildirim, Oktay, OQ, 0T, 23

Yoshihara, Kosuke, 29

Yoshitake, Shusuke, 10, 1Q

Yu, Jengyi, 19

Zahedmanesh, Houman, OL

Zdravkov, Alexander, OD

Zhang, Yu, 25

Zhang, Yunqiang, $1 \mathrm{C}$

Zhou, Xibin, 1C 


\title{
Conference Committee
}

\author{
Symposium Chair
}

Bruce W. Smith, Rochester Institute of Technology (United States)

Symposium Co-Chair

Will Conley, Cymer, An ASML Company (United States)

Conference Chair

Eric M. Panning, Intel Corporation (United States)

Conference Co-Chair

Kenneth A. Goldberg, Lawrence Berkeley National Laboratory (United States)

Conference Program Committee

Markus Bender, Advanced Mask Technology Center GmbH Company KG (Germany)

Jos P. Benschop, ASML Netherlands B.V. (Netherlands)

Robert L. Brainard, SUNY CNSE/SUNYIT (United States)

Martin Burkhardt, IBM Thomas J. Watson Research Center (United States)

Deniz Elizabeth Civay, GLOBALFOUNDRIES Inc. (United States)

Daniel A. Corliss, IBM Corporation (United States)

Yasin Ekinci, Paul Scherrer Institut (Switzerland)

Nelson M. Felix, IBM Corporation (United States)

Emily E. Gallagher, IMEC (Belgium)

Florian Gstrein, Intel Corporation (United States)

Naoya Hayashi, Dai Nippon Printing Company, Ltd. (Japan)

Eric Hendrickx, IMEC (Belgium)

Soichi Inove, Toshiba Corporation (Japan)

Bryan S. Kasprowicz, Photronics, Inc. (United States)

Insung Kim, SAMSUNG Electronics Company, Ltd.

(Korea, Republic of)

Seong-Sue Kim, SAMSUNG Electronics Company, Ltd.

(Korea, Republic of)

Ted Liang, Intel Corporation (United States)

Chang-Moon Lim, SK Hynix, Inc. (Korea, Republic of)

Anna Lio, Intel Corporation (United States)

Lawrence S. Melvin III, Synopsys, Inc. (United States) 
Hiroaki Morimoto, Toppan Printing Company, Ltd. (Japan)

Patrick P. Naulleau, Lawrence Berkeley National Laboratory

(United States)

Christopher S. Ngai, Applied Materials, Inc. (United States)

Shinji Okazaki, Gigaphoton Inc. (Japan)

Jan Hendrik Peters, Carl Zeiss SMS GmbH (Germany)

Jorge J. Rocca, Colorado State University (United States)

Kurt G. Ronse, IMEC (Belgium)

Tsutomu Shoki, HOYA Corporation (Japan)

Akiyoshi Suzuki, Gigaphoton Inc. (Japan)

Anna Tchikoulaeva, Lasertec U.S.A., Inc. Zweigniederlassung

Deutschland (Germany)

Thomas I. Wallow, ASML Brion (United States)

Obert R. Wood II, GLOBALFOUNDRIES Inc. (United States)

Anthony Yen, Taiwan Semiconductor Manufacturing Company Ltd. (Taiwan, China)

\section{Session Chairs}

1 Keynote Session

Eric M. Panning, Intel Corporation (United States)

Kenneth A. Goldberg, Lawrence Berkeley National Laboratory

(United States)

2 EUV Materials I: Metal-Based EUV Resists: Joint Session with Conferences 10146 and 10143

Thomas I. Wallow, ASML Brion (United States)

Robert L. Brainard, SUNY CNSE/SUNYIT (United States)

3 EUV Materials II: Fundamentals I: Joint Session with Conferences 10146 and 10143

Clifford L. Henderson, Georgia Institute of Technology (United States)

Anna Lio, Intel Corporation (United States)

4 Tutorial Session: Joint Session with Conferences 10143 and 10146

Eric M. Panning, Intel Corporation (United States)

Kenneth A. Goldberg, Lawrence Berkeley National Laboratory

(United States)

5 Integration

Jos P. Benschop, ASML Netherlands B.V. (Netherlands)

Daniel A. Corliss, IBM Corporation (United States) 
6 EUV Mask Inspection and Imaging: Joint Session with Conferences 10143 and 10145

Anna Tchikoulaeva, Lasertec U.S.A., Inc. Zweigniederlassung Deutschland (Germany)

Ofer Adan, Applied Materials, Ltd. (Israel)

7 EUV Optics and Pellicle

Patrick P. Naulleau, Lawrence Berkeley National Laboratory (United States)

Yasin Ekinci, Paul Scherrer Institut (Switzerland)

9 Resist Advances and Integration

Robert Bristol, Intel Corporation (United States)

Nelson M. Felix, IBM Corporation (United States)

10 Resist Modeling

Thomas I. Wallow, ASML Brion (United States)

Christopher S. Ngai, Applied Materials, Inc. (United States)

11 Masks I

Emily E. Gallagher, IMEC (Belgium)

Obert R. Wood II, GLOBALFOUNDRIES Inc. (United States)

12 Masks II

Malahat Tarassasoli, Intel Corporation (United States)

Naoya Hayashi, Dai Nippon Printing Company, Ltd. (Japan)

13 Patterning I

Soichi Inove, Toshiba Corporation (Japan)

Bryan S. Kasprowicz, Photronics, Inc. (United States)

14 Patterning II

Martin Burkhardt, IBM Thomas J. Watson Research Center

(United States)

Thomas Scherise, Carl Zeiss SMT GmbH (Germany)

15 Source

Akiyoshi Suzuki, Gigaphoton Inc. (Japan)

Jos P. Benschop, ASML (United States) 
Proc. of SPIE Vol. 10143 1014301-16 Downloaded From: https://www.spiedigitallibrary.org/conference-proceedings-of-spie on 26 Apr 2023
Terms of Use: https://www.spiedigitallibrary.org/terms-of-use 